\title{
Contribution to the local thermophysical characterization of murals paintings of the inheritance by stimulated infra-red thermography
}

\author{
By K. Mouhoubi ${ }^{\star}$, J.L Bodnar ${ }^{\star}$, J.L. Nicolas ${ }^{\star}$, V. Detalle ${ }^{\star \star}$, J.M. Vallet $^{\star \star \star}$, T. Duvaut $^{\star}$
}

\author{
* GRESPI / ECATHERM, UFR Sciences Exactes et Naturelles, BP 1039, 51687 Reims. \\ ${ }^{*}$ LRMH, 29 avenue du Paris, 77420 Champs sur Marne. \\ ${ }^{\star * \star}$ CICRP, 21 rue Guibal, 13003 Marseille.
}

\begin{abstract}
The object of this work is to approach the possibilities of stimulated infrared thermography for in situ measurement of thermal diffusivity of mural paintings of the inheritance.

We present first the principle of the method. We show then the feasibility of the measurement, using theoretical simulations.

Finally, we show that the method allows a good estimation of the thermal diffusivity of a partial copy of the "Saint Christophe" of the Campana collection in the Louvre.
\end{abstract}

\section{Introduction}

Conservation of cultural heritage is essential. For this reason, many physicals methods are already implemented by the restorers of the inheritance. Without being exhaustive, we can cite the optical analysis, the $X$ analysis or the acoustic analysis [1-4]. These techniques are not, of course, universal. It explains why other modes of non destructive testing are still studied in the research laboratories. Stimulated infrared thermography is one of these new methods. This technique is promising. It already showed its possibilities for the detection of delamination located in mural painting of the inheritance [5-18]. It already allowed, for example, the in situ analysis, of the "Saint Christophe" of the "Campana" collection in the "Louvre", the in situ analysis of the "Saint Martin " of the "Saint Florentin" church in "Bonnet", the in situ analysis of the "Traversée du désert" of the painted ceilings of the "Savin Saint sur Gartempe" abbey (classified with the world inheritance of UNESCO), the in situ analysis of the " Saint Pierre livré aux soldats de Pilâtes " of the "Saint Pierre" church of "Villefranche sur mer" or the in situ analysis of the painted ceilings of the "Médicis" library of the French Senate [12-18].

The possibilities of the stimulated infra-red thermography for the detection of delamination located in murals paintings of the inheritance are then now recognized by the scientific community. There is now to study its possibilities for the dimensional characterization of theses defects. Our team already proposed two approaches allowing a local and in situ measurement of the thermal diffusivity parameter [19-20]. Having the objective to determine, the best measurement protocol (the most robust, the most reliable, the less intrusive), we still study other ways of analyses. The work presented here comes within this framework. It presents a third way to measure the local thermal diffusivity of a work of art. It is based on the temporal analysis of the maximum of the photothermal signal, measured at the place of a laser excitation.

Our presentation is divided into three parts:

We present first the principle of this new measurement method.

We show then the feasibility of the approach using theoretical simulations.

Finally, we shows experimentally, that the method allows a good and in situ estimation of the thermal diffusivity of a partial copy of the "Saint Christophe" of the "Campana" collection in the Louvre.

\section{Longitudinal thermal diffusivity measuring method developed for the study}

The longitudinal thermal diffusivity measuring method principle developed for the study is the following. A sample is submitted on its front face to a laser excitation at a given position. It is temporally close to a Dirac delta function $\delta(t)$. It is spatially close to a Gaussian function. Variations in the temperature field induced by the excitation are measured with an infrared camera of thermography. Setting off from the time change of this temperature field, the 
material's thermal diffusivity value is inferred, thanks to a mathematical post-treatment. This mathematical posttreatment, which this measuring technique is based on, must be looked at closely.

Let $L$ be a plate's thickness, radially semi-infinite. Let a thermal disturbance be very short (temporal Dirac delta function $\delta(t)$ ) and spatially of a Gaussian shape. This excitation is applied to $t=0$ in the centre of the previous plate. Let $\mathrm{R}$ be the radius beam of the excitation spot (measured at $\mathrm{Q}_{\max } / \mathrm{e}^{2}$, with $\mathrm{Q}_{\max }$ the energy value in the centre of the excitation spot). Let $\lambda, \rho, \mathrm{c}$ and a be, respectively, thermal conductivity, density, heat capacity and thermal diffusivity of the studied sample. Initially, the sample is in temperature balance within the environment. The temperature of this one is equal to $T_{\text {ext. }}$ Last, the model does not take into account the convecto-radiative exchanges between the studied sample and the environment. The mathematical translation of these assumptions leads to the following differential system:

$$
\begin{gathered}
\Delta T(r, z, t)=\frac{1}{a} \cdot \frac{\partial T(r, z, t)}{\partial t} \\
\mathrm{Z}=0:-\lambda \frac{\partial T(z, 0, t)}{\partial t}=\frac{2 Q}{\pi R^{2}} \operatorname{Exp}\left(-\frac{2 r^{2}}{R^{2}}\right) \delta(t) \\
Z=L:-\lambda \frac{\partial T(z, L, t)}{\partial t}=0 \\
t=0: T=T_{\text {ext }}
\end{gathered}
$$

The resolution of this differential system was already presented in [20]. To resolve this differential system, two integral transformations are chosen: On one hand Hankel's transformation of 0 order on $r$ axis; On the other, a Fourier transformation in time. It leads to the space-time evolution of the temperature in front face of the studied sample given by the formula (2).

$$
T(r, 0, t) \approx \frac{2 Q}{b \sqrt{\pi^{3} t}} \cdot \frac{1}{R^{2}+8 a t} \cdot \exp \left(-\frac{2 r^{2}}{R^{2}+8 a t}\right)
$$

Now let us consider the maximum value of the temperature. It is obtained in the center of Gaussian, i.e. in $r=0$

$$
T_{\max }(0,0, t) \approx \frac{2 Q}{b \sqrt{\pi^{3} t}} \cdot \frac{1}{R^{2}+8 a t}
$$

By rearranging this expression mathematically, we can then write:

$$
\frac{1}{T_{\max }(0,0, t) \sqrt{t}} \approx \frac{b R^{2} \sqrt{\pi^{3}}}{2 Q}\left(1+\frac{8 a}{R^{2}} t\right)
$$


Formula (4) shows that required thermal diffusivity can thus be simply calculate from the slope, the origin ordinate, of the inverse of the product of the maximum temperature of the thermal spot generated by the laser excitation and the square root of time (5). That thus offers to us a new method of estimate of this thermophysical parameter.

$$
a=\frac{\text { slope. } R^{2}}{\text { 8.ordinate }}
$$

\section{Theoretical simulations}

To test this new method of longitudinal thermal diffusivity measurement, we have developed simulations. They use the finite element method to solve the preceding differential system (1). The simulations conditions retained for the study are the following: First, we considered a 3D geometry. Then, we considered a plaster block. It indeed presents thermophysical properties close to those of a mural painting. We considered a thermal conductivity equal to $\lambda=0,4$ $\mathrm{W} / \mathrm{mK}$, a density equal to $\rho=1100 \mathrm{Kg} / \mathrm{m}^{3}$, and a heat capacity equal to $\mathrm{c}=830 \mathrm{~J} / \mathrm{Kg} \mathrm{K}$. The thermal diffusivity is then equal to $\mathrm{a}=4,38 \cdot 10^{-7} \mathrm{~m}^{2} / \mathrm{s}$. By simplicity, the shape of this plaster block was taken rectangular. Its dimensions are the following: Its length is equal to $12 \mathrm{~cm}$. Its width is equal to $12 \mathrm{~cm}$. And its thickness is equal to $3 \mathrm{~mm}$. The excitation, with a Gaussian shape was deposited in the center of this plaster block. Its characteristics are the following: Its power is equal to 3 Watts (it is the maximum power delivered by the laser of the laboratory associated with this study). To approach a Dirac function, the duration of excitation is equal to $20 \mathrm{~ms}$. The characteristic radius of the excitation is equal to $660 \mu \mathrm{m}$. It is a classical value accessible by the experiment. Finally, to reduce the computing times, we considered a progressive meshing of the studied sample. It is finer at the place of the laser excitation and with a greater width elsewhere. From the results provided by these simulations, we extracted, at each moment, the maximum value of the temperature, measured at the place of the laser excitation. We then plotted the temporal evolution of the inverse of the product: maximum temperature of the thermal spot generated by the laser excitation multiplied by the square root of time (figure 1). We then estimated the slope and the origin ordinate of the line obtained. We then used formula 5 to determine required thermal diffusivity. In our study, figure 1, shows a slope equal to $0,113 \mathrm{~K}^{-1} \mathrm{~s}^{-3 / 2}$ and one origin ordinate equal to $1,4010^{-2} \mathrm{~K}^{-1} \mathrm{~s}^{-}$ $1 / 2$. These values lead to a thermal diffusivity equal to $4,39.10^{-7} \mathrm{~m}^{2} / \mathrm{s}$. The theoretical value is equal to $4,38.10^{-7} \mathrm{~m}^{2} / \mathrm{s}$. These values are very close. It shows the feasibility of the method.

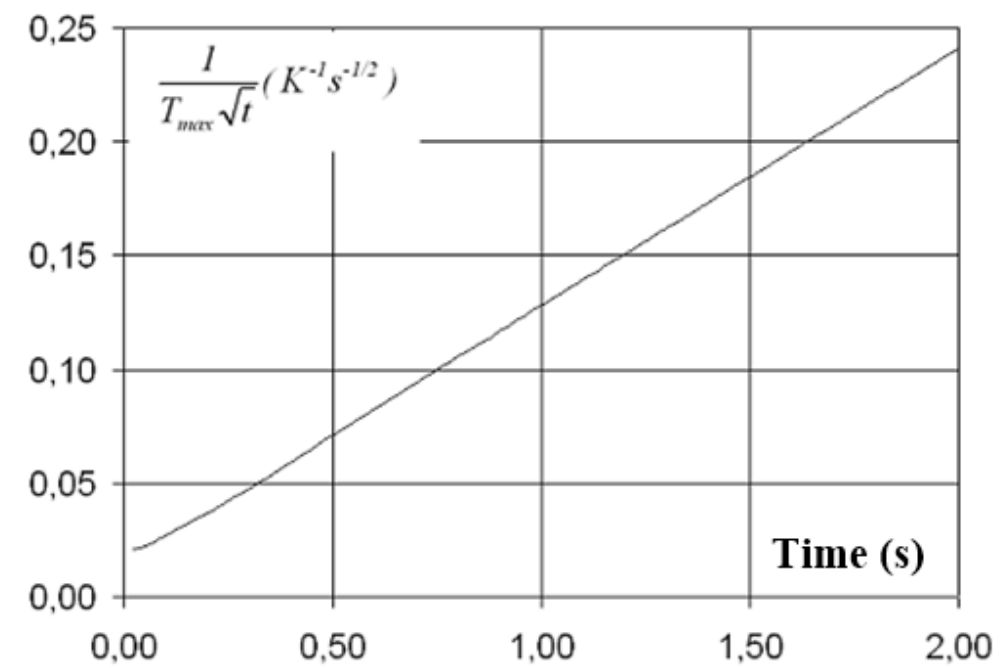

Fig 1.: Temporal evolution of the inverse of the product maximum temperature of the thermal signature generated by the laser excitation multiplied by the square root of time 


\section{Experimental study}

\subsection{The experimental device implemented}

The results obtained during the theoretical study are encouraging. Then we passed in a second time to an experimental study. The experimental device implemented for the study is the SAMMTHIR system. It is a device of our laboratory. The excitation source is a laser diode. Its wavelength is equal to $810 \mathrm{~nm}$. It is coupled with collimation and focusing optics. The acquisition system is micro bolometers infrared camera. To obtain a sufficient space resolution, it works in macro mode. The latter is placed perpendicularly with the sample, at a distance equal about to $5 \mathrm{~cm}$. Because of the obstruction of the camera, the laser spot lighted in a tilted way the studied sample. Its shape is then slightly elliptic. The power of the laser spot is equal to $2 \mathrm{~W}$. The duration of the excitation is equal to $20 \mathrm{~ms}$. The acquisition frequency of the infra-red camera thermography is equal to $50 \mathrm{~Hz}$.

\subsection{Study of a mural painting}

The studied sample is a mural painting. It is a partial copy of "Saint Christophe" of the Campana collection in the Louvre (figure 2). It was already characterized previously using two methods: On the one hand, it was characterized by space Fourier analysis of the photothermal response of the sample submitted to a localised laser excitation [19]. On the other hand, it was characterized by a temporal analysis of the characteristic diameter of the photothermal signature of the laser spot [20]. In the first case the local thermal diffusivity estimated at the place of the right eye of the Jesus child was equal to $5,1310^{-7} \mathrm{~m}^{2} \mathrm{~s}^{-1}$. In the second case and in the same place, the estimated thermal diffusivity was equal $5,0910^{-7} \mathrm{~m}^{2} \mathrm{~s}^{-1}$.

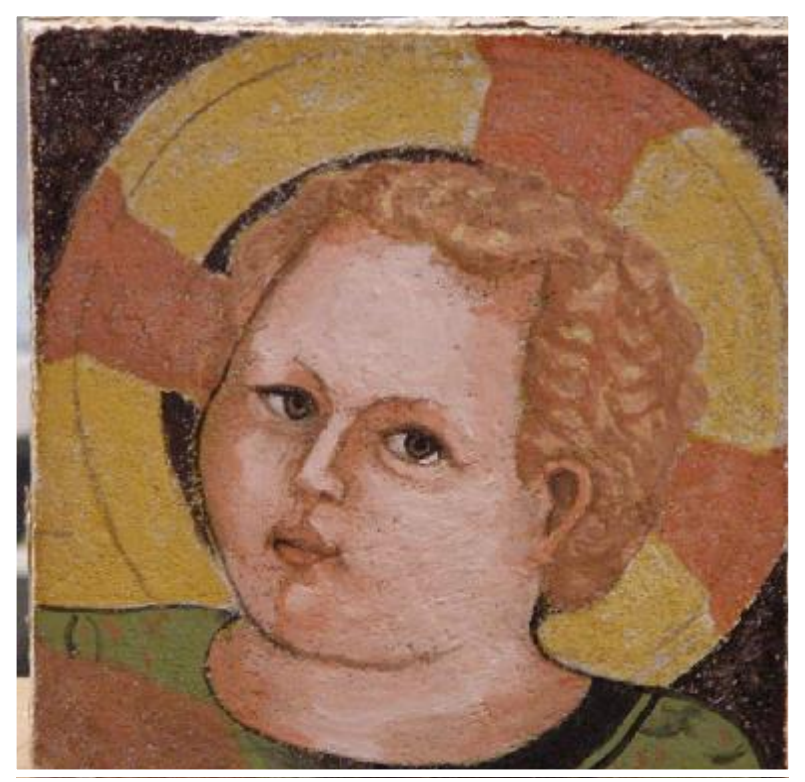

Fig 2: The studied mural painting

It is always with this position that we developed our new analysis. We used the same analyze protocol as at the time of the theoretical study. We obtained the result presented in figure 3 . This figure shows, as waited, a line with a positive slope. The value of this slope is equal to $37,610^{-3} \mathrm{~K}^{-1} \mathrm{~s}^{-3 / 2}$. The value of the origin ordinate origin is equal to 9,5 $10^{-3} \mathrm{~K}^{-1} \mathrm{~s}^{-1 / 2}$. The first thermogram gives an excitation characteristic radius equal to $1,01 \mathrm{~mm}$. Formula (5) leads then to a thermal diffusivity equal to $4,9610^{-7} \mathrm{~m} \mathrm{~S} \mathrm{~S}^{-1}$. This experimental value is close to the previous values. It confirms the feasibility of the method. 


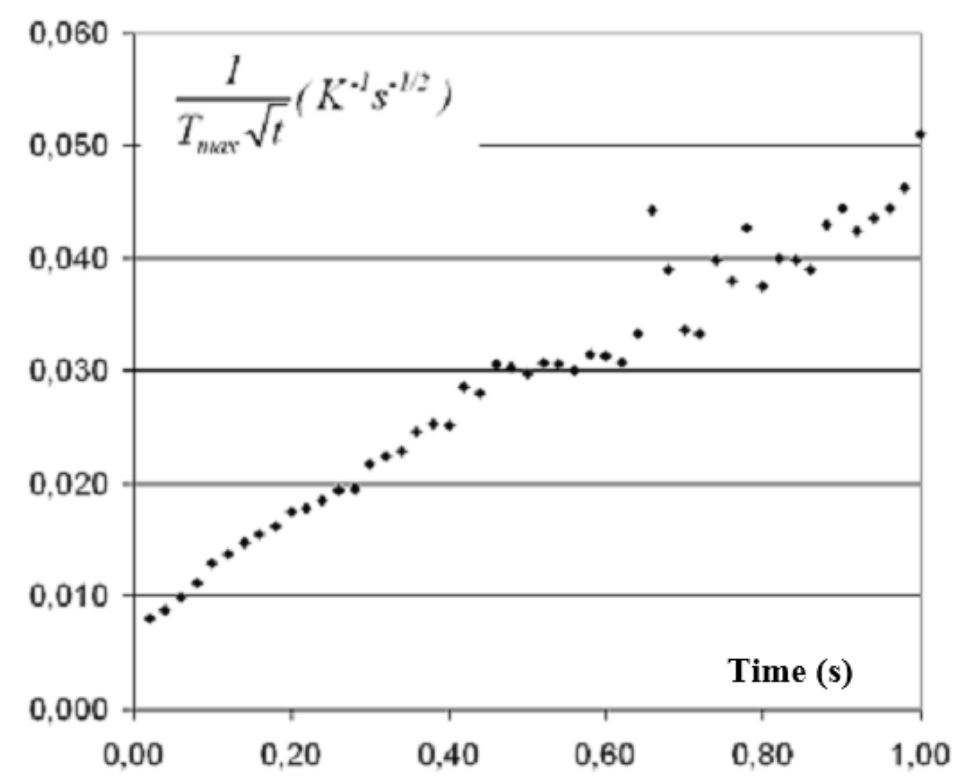

Fig 3.: Characterization of a mural painting using SAMMTHIR system

\section{Conclusion}

In this work, we studied the possibilities of stimulated infra-red thermography for the measurement of longitudinal thermal diffusivity of mural painting.

First we presented the principle of the measurement method. It consists to study the temporal evolution of the maximum value of the photothermal signal, measured at the place of a laser excitation.

We presented then the simulations developed for the study and showed theoretically that the photothermal method allowed the measurement of the longitudinal thermal diffusivity of a plaster sample.

The theoretical results obtained being positive, we developed in a third step, an experimental study. We then presented the experimental device developed for the study.

Finally we showed in a experimental way, that the method permits a good estimate of the longitudinal thermal diffusivity of a fresco.

These theoretical and experimental results are encouraging since open. They seem open the way to an in situ photothermal characterization of works of art. They have now to be generalized and be implemented during the restoration of works of art. Studies going in this direction are in progress.

\section{REFERENCES}

[1] W.Eposti, S. Levrero, G.Stella, actes de la 1ère conférence internationale sur les essais non destructifs en conservation d'œuvres d'art, Rome, 1985, p. I/11.1-1/11.14.

[2] V. Tornari, E. Esposito, V. Zafiropulos, N. Paone, C. Fotakis, E.P. Tomasini, N. Smyrmakis, S. Stassinopulos, M. Stefanaggi, «Non-invasive laser mesurement for diagnosing the state of conservation of frescoes and wooden icons ", 4th European Commission Conference on Researchfor protection, conservation and enhancement of cultural heritage : Oppotunities for European enterprises, Strasbourg, 2000, p. 74-80.

[3] T. Fricke-Begemann, G. Gülker, K.D. Hinsch, H. Joost, „Remote localisation of debonded areas in historical murals by TV-holography“1999, p. 247-255; Schirripa Spagnolo, Guattari, Grinzato, 1999.

[4] P. Baratono, F. Cardelli, M. Diana,C. Trombetta, G. Venturini : Termografia e analisi di immagine, ultime applicazioni su strutture architettoniche, $2^{\circ}$ Conferenza internazionale sulle prove non distruttive, metodi e indagini ambientali per lo studio e la conservazione delle opere d'arte, Pérouse, 1988, p. 1/9.1-1/9.13

[5] E. Grinzato, P.G. Bison, S. Marinetti, V. Vavilov, : Active thermal testing of delaminations in frescoes' plaster, 4th International Conference on Non-destructive Testing of Works of Art, Berlin,1994, p. 357-367 
[6] E. Grinzato, P.G. Bison, S. Marinetti, V. Vavilov, : Non-destructive evaluation of delamination in fresco plaster using transient infrared thermography. Research in NDE, vol 5, n4, pp257-274, 1994.

[7] G. Schirripa Spagnolo, G. Guattari, E. Grinzato : Frescoes Diagnostics by electro-optic holography and infrared thermography. Proc I the 6th World Conference on NDT and Microanalysis in Diagnostics and Conservation of Cultural and Environnemental Heritage, Rome, 1999, p. 385-398.

[8] E. Grinzato, P.G. Bison, S. Marinetti: Monitoring of ancient buildings by the thermal method. Journal of Cultural Heritage 3 (2002) 21-29.

[9] E. Grinzato, C. Bressan, S. Marinetti, P. Bison, C. Bonacina: Monitoring of the Scrovegni chapel by IR thermography: Giotto at infrared, Infrared physics and technology, vol 43, n³-5, 2002, pp165-169.

[10] G. Carlomagno, C. Meola: Comparison between thermographic techniques for frescoes NDT, NDT\&E International 35 (2002) 559-565.

[11] N. Avdelidis, A. Moropoulou: Applications of infrared thermography for the investigation of historic structures, Journal of Cultural Heritage 5 (2004) 119-127.

[12] G. Szatanik : Etude et restauration d'une peinture murale représentant Saint Christophe (collection Campana, Louvre). Essai d'application de la thermographie infrarouge stimulée pour l'examen du support. INP, Sept 2004.

[13] J.C. Candoré : Détection et caractérisation de défauts par thermographie infrarouge stimulée : Application au contrôle d'œuvres d'art. PhD, Reims, June 2010.

[14] G. Szatanik, M. Gerard, J.L.Bodnar : La thermographie infrarouge stimulée pour l'examen des enduits dans les peintures murales: Application au Saint Christophe portant l'enfant Jésus. Journal de Conservation et restauration (coré), $\mathrm{n}^{\circ} 17$, décembre 2006, pp $64-70$.

[15] J. C. Candoré, J.L Bodnar, V. Detalle, P. Grossel : Nondestructive testing of work of art by stimulated infrared thermography. European physical journal Applied Physics, 2012, vol 57, 02, pp 21002 -210011.

[16] J.L. Bodnar, J.L. Nicolas, J.C. Candoré, V. Detalle: Non-destructive testing by infrared thermography under random excitation and ARMA analysis. International journal of thermo physics, DOI 10.1007/s10765-012$1265-2$.

[17] J.L Bodnar, J. C Candoré, J.L. Nicolas, G. Szatanik, V. Detalle, J.M.Vallet : Stimulated infrared thermography applied to help for restoration of mural paintings, NDT/E International, 49, pp 40-46, 2012

[18] J.L. Bodnar, K. Mouhoubi, G. Szatanik-Perrier, J.M. Vallet and V. Detalle: Photothermal thermography applied to the non-destructive testing of different types of works of art, International Journal of Thermophysics, DOI 10.1007/s10765-012-1301-2.

[19] J.C. Candoré, J.L Bodnar, V. Detalle, P. Grossel : Characterization of defects situated in a fresco by stimulated infrared thermography, European physical journal Applied Physics, 2012, vol 57, 01 , pp 11002 11008

[20] J.L Bodnar, J.L. Nicolas, K. Mouhoubi, V. Detalle: Stimulated infrared thermography applied to thermophysical characterization of cultural heritage mural paintings, 2012, 60, 21003 DOI : 10.1051/epjap/2012120280 